\title{
Generalized common fixed point theorems in complex valued metric spaces and applications
}

\author{
Wutiphol Sintunavarat and Poom Kumam*
}

* Correspondence: poom. kum@kmutt.ac.th

Department of Mathematics,

Faculty of Science, King Mongkut's University of Technology Thonburi (KMUTT), Bangkok 10140, Thailand

\begin{abstract}
Recently, Azam et al. introduced new spaces called the complex valued metric spaces and established the existence of fixed point theorems under the contraction condition. In this article, we extend and improve the condition of contraction of the results of Azam et al. and also apply the main result to the unique common solution of system of Urysohn integral equation.

Mathematics Subject Classification (2000): 47H09; 47H10.
\end{abstract}

Keywords: complex valued metric spaces, fixed points, common fixed points, weakly compatible mappings

\section{Introduction}

Fixed point theory became one of the most interesting area of research in the last fifty years for instance research about optimization problem, control theory, differential equations, economics, and etc. The fixed point theorem, generally known as the Banach contraction mapping principle, appeared in explicit form in Banach's thesis in 1922 [1]. Since its simplicity and usefulness, it became a very popular tool in solving many problems in mathematical analysis. Later, a number of articles in this field have been dedicated to the improvement and generalization of the Banach's contraction mapping principle in several ways in many spaces (see [2-17]).

In the other hand, the study of metric spaces expressed the most important role to many fields both in pure and applied science such as biology, medicine, physics, and computer science (see $[18,19]$ ). Many authors generalized and extended the notion of a metric spaces such as a vector-valued metric spaces of Perov [20], a G-metric spaces of Mustafa and Sims [21], a cone metric spaces of Huang and Zhang [22], a modular metric spaces of Chistyakov [23], and etc.

Recently, Azam et al. [24] first introduced the complex valued metric spaces which is more general than well-know metric spaces and also gave common fixed point theorems for mappings satisfying generalized contraction condition.

Theorem 1.1 (Azam et al. [24]). Let $(X, d)$ be a complete complex valued metric space and $S, T: X \rightarrow X$. If $S$ and $T$ satisfy

$$
d(S x, T y) \precsim \lambda d(x, y)+\frac{\mu d(x, S x) d(y, T y)}{1+d(x, y)}
$$


for all $x, y \in X$, where $\lambda, \mu$ are nonnegative reals with $\lambda+\mu<1$. Then $S$ and $T$ have a common fixed point.

The aim of this article is to extend and improve the conditions of contraction of this theorem from the constant of contraction to some control functions and establish the common fixed point theorems which are more general than the result of Azam et al. [24] and also give the results for weakly compatible mappings in complex valued metric spaces. As applications, we claim that the existence of common solution of system of Urysohn integral equation by using our results.

\section{Preliminaries}

Let $\mathbb{C}$ be the set of complex numbers and $z_{1}, z_{2} \in \mathbb{C}$. Define a partial order $\preccurlyeq$ on $\mathbb{C}$ as follows:

$$
z_{1} \precsim z_{2} \text { if and only if } \operatorname{Re}\left(z_{1}\right) \leq \operatorname{Re}\left(z_{2}\right) \text { and } \operatorname{Im}\left(z_{1}\right) \leq \operatorname{Im}\left(z_{2}\right)
$$

that is $z_{1} \precsim z_{2}$ if one of the following holds

(C1): $\operatorname{Re}\left(z_{1}\right)=\operatorname{Re}\left(z_{2}\right)$ and $\operatorname{Im}\left(z_{1}\right)=\operatorname{Im}\left(z_{2}\right)$;

(C2): $\operatorname{Re}\left(z_{1}\right)<\operatorname{Re}\left(z_{2}\right)$ and $\operatorname{Im}\left(z_{1}\right)=\operatorname{Im}\left(z_{2}\right)$;

(C3): $\operatorname{Re}\left(z_{1}\right)=\operatorname{Re}\left(z_{2}\right)$ and $\operatorname{Im}\left(z_{1}\right)<\operatorname{Im}\left(z_{2}\right)$;

(C4): $\operatorname{Re}\left(z_{1}\right)<\operatorname{Re}\left(z_{2}\right)$ and $\operatorname{Im}\left(z_{1}\right)<\operatorname{Im}\left(z_{2}\right)$.

In particular, we will write $z_{1} \lessgtr z_{2}$ if $z_{1} \neq z_{2}$ and one of (C2), (C3), and (C4) is satisfied and we will write $z_{1} \prec z_{2}$ if only (C4) is satisfied.

Remark 2.1. We obtained that the following statements hold:

(i) $a, b \in \mathbb{R}$ and $a \leq b \Rightarrow a z \preceq b z \forall z \in \mathbb{C}$.

(ii) $0 \preceq z_{1} \preccurlyeq z_{2} \Rightarrow\left|z_{1}\right|<\left|z_{2}\right|$,

(iii) $z_{1} \preccurlyeq z_{2}$ and $z_{2} \prec z_{3} \Rightarrow z_{1} \prec z_{3}$.

Definition 2.2 ([24]). Let $X$ be a nonempty set. Suppose that the mapping $d: X \times X$ $\rightarrow \mathbb{C}$ satisfies the following conditions:

(i) $0 \preceq d(x, y)$, for all $x, y \in X$ and $d(x, y)=0$ if and only if $x=y$;

(ii) $d(x, y)=d(y, x)$ for all $x, y \in X$;

(iii) $d(x, y) \lessgtr d(x, z)+d(z, y)$, for all $x, y, z \in X$.

Then $d$ is called a complex valued metric on $X$ and $(X, d)$ is called a complex valued metric space.

Example 2.3. Let $X=\mathbb{C}$. Define the mapping $d: X \times X \rightarrow \mathbb{C}$ by

$$
d\left(z_{1}, z_{2}\right)=e^{i k}\left|z_{1}-z_{2}\right|
$$

where $k \in \mathbb{R}$. Then $(X, d)$ is a complex valued metric space.

Definition 2.4 ([24]). Let $(X, d)$ be a complex valued metric space.

(i) A point $x \in X$ is called interior point of a set $A \subseteq X$ whenever there exists $0 \prec r$

$\in \mathbb{C}$ such that 


$$
B(x, r):=\{y \in X \mid d(x, y) \prec r\} \subseteq A .
$$

(ii) A point $x \in X$ is called a limit point of $A$ whenever for every $0 \prec r \in \mathbb{C}$,

$$
B(x, r) \cap(A-X) \neq \emptyset \text {. }
$$

(iii) A subset $A \subseteq X$ is called open whenever each element of $A$ is an interior point of $A$.

(iv) A subset $A \subseteq X$ is called closed whenever each limit point of $A$ belongs to $A$.

(v) A sub-basis for a Hausdorff topology $\tau$ on $X$ is a family

$$
F=\{B(x, r) \mid x \in X \text { and } 0 \prec r\} .
$$

Definition $2.5([24])$. Let $(X, d)$ be a complex valued metric space, $\left\{x_{n}\right\}$ be a sequence in $X$ and $x \in X$.

(i) If for every $c \in \mathbb{C}$, with $0 \prec c$ there is $N \in \mathbb{N}$ such that for all $n>N, d\left(x_{n}, x\right) \prec c$, then $\left\{x_{n}\right\}$ is said to be convergent, $\left\{x_{n}\right\}$ converges to $x$ and $x$ is the limit point of $\left\{x_{n}\right\}$. We denote this by $\lim _{n \rightarrow \infty} x_{n}=x$ or $\left\{x_{n}\right\} \rightarrow x$ as $n \rightarrow \infty$.

(ii) If for every $c \in \mathbb{C}$, with $0 \prec c$ there is $N \in \mathbb{N}$ such that for all $n>N, d\left(x_{n}, x_{n+m}\right)$ $\prec c$, where $m \in \mathbb{N}$, then $\left\{x_{n}\right\}$ is said to be Cauchy sequence.

(iii) If every Cauchy sequence in $X$ is convergent, then $(X, d)$ is said to be a complete complex valued metric space.

Lemma 2.6 ([24]). Let $(X, d)$ be a complex valued metric space and let $\left\{x_{n}\right\}$ be a sequence in $X$. Then $\left\{x_{n}\right\}$ converges to $x$ if and only if $\left|d\left(x_{n}, x\right)\right| \rightarrow 0$ as $n \rightarrow \infty$.

Lemma 2.7 ([24]). Let $(X, d)$ be a complex valued metric space and let $\left\{x_{n}\right\}$ be a sequence in $X$. Then $\left\{x_{n}\right\}$ is a Cauchy sequence if and only if $\left|d\left(x_{n}, x_{n+m}\right)\right| \rightarrow 0$ as $n \rightarrow$ $\infty$, where $m \in \mathbb{N}$.

Here, we give some notions in fixed point theory.

Definition 2.8. Let $S$ and $T$ be self mappings of a nonempty set $X$.

(i) A point $x \in X$ is said to be a fixed point of $\mathrm{T}$ if $T x=x$.

(ii) A point $x \in X$ is said to be a coincidence point of $S$ and $T$ if $S x=T x$ and we shall called $w=S x=T x$ that a point of coincidence of $S$ and $T$.

(iii) A point $x \in X$ is said to be a common fixed point of $S$ and $T$ if $x=S x=T x$.

In 1976, Jungck [25] introduced concept of commuting mappings as follows:

Definition 2.9 ([25]). Let $X$ be a non-empty set. The mappings $S$ and $T$ are commuting if

$$
T S x=S T x
$$

for all $x \in X$. 
Afterward, Sessa [26] introduced concept of weakly commuting mappings which are more general than commuting mappings as follows:

Definition 2.10 ([26]). Let $S$ and $T$ be mappings from a metric space $(X, d)$ into itself. The mappings $S$ and $T$ are said to be weakly commuting if

$$
d(S T x, T S x) \leq d(S x, T x)
$$

for all $x \in X$.

In 1986, Jungck [27] introduced the more generalized commuting mappings in metric spaces, called compatible mappings, which also are more general than the concept of weakly commuting mappings as follows:

Definition 2.11 ([27]). Let $S$ and $T$ be mappings from a metric space $(X, d)$ into itself. The mapping $S$ and $T$ are said to be compatible if

$$
\lim _{n \rightarrow \infty} d\left(S T x_{n}, T S x_{n}\right)=0
$$

whenever $\left\{x_{n}\right\}$ is a sequence in $X$ such that $\lim _{n \rightarrow \infty} S x_{n}=\lim _{n \rightarrow \infty} T x_{n}=z$ for some $z$ $\in X$.

Remark 2.12. In general, commuting mappings are weakly commuting and weakly commuting mappings are compatible, but the converses are not necessarily true and some examples can be found in [25,27-29].

In 1996, Jungck introduced the concept of weakly compatible mappings as follows:

Definition 2.13 ([30]). Let $S$ and $T$ be self mappings of a nonempty set $X$. The mapping $S$ and $T$ are weakly compatible if $S T x=T S x$ whenever $S x=T x$.

We can see an example to show that there exists weakly compatible mappings which are not compatible mappings in metric spaces in Djoudi and Nisse [31].

The following lemma proved by Haghi et al. [32] is useful for our main results:

Lemma 2.14 ([32]). Let $X$ be a nonempty set and $T: X \rightarrow X$ be a function. Then there exists a subset $E \subseteq X$ such that $T(E)=T(X)$ and $T: E \rightarrow X$ is one-to-one.

\section{Main results}

Theorem 3.1. Let $(X, d)$ be a complete complex valued metric space and $S, T: X \rightarrow X$. If there exists a mapping $\Lambda, \Xi: X \rightarrow[0,1)$ such that for all $x, y \in X$ :

(i): $\Lambda(S x) \leq \Lambda(x)$ and $\Xi(S x) \leq \Xi(x)$;

(ii): $\Lambda(T x) \leq \Lambda(x)$ and $\Xi(T x) \leq \Xi(x)$;

(iii): $(\Lambda+\Xi)(x)<1$;

(iv): $d(S x, T y) \precsim \Lambda(x) d(x, y)+\frac{\Xi(x) d(x, S x) d(y, T y)}{1+d(x, y)}$.

Then $S$ and $T$ have a unique common fixed point.

Proof. Let $x_{0}$ be an arbitrary point in $X$. Since $S(X) \subseteq X$ and $T(X) \subseteq X$, we can construct the sequence $\left\{x_{k}\right\}$ in $X$ such that

$$
x_{2 k+1}=S x_{2 k} \text { and } x_{2 k+2}=T x_{2 k+1}
$$


for all $k \geq 0$. From hypothesis and (3.1) we get

$$
\begin{aligned}
d\left(x_{2 k+1}, x_{2 k+2}\right) & =d\left(S x_{2 k}, T x_{2 k+1}\right) \\
& \precsim \Lambda\left(x_{2 k}\right) d\left(x_{2 k}, x_{2 k+1}\right)+\frac{\Xi\left(x_{2 k}\right) d\left(x_{2 k}, S x_{2 k}\right) d\left(x_{2 k+1}, T x_{2 k+1}\right)}{1+d\left(x_{2 k}, x_{2 k+1}\right)} \\
& =\Lambda\left(x_{2 k}\right) d\left(x_{2 k}, x_{2 k+1}\right)+\frac{\Xi\left(x_{2 k}\right) d\left(x_{2 k}, x_{2 k+1}\right) d\left(x_{2 k+1}, x_{2 k+2}\right)}{1+d\left(x_{2 k}, x_{2 k+1}\right)} \\
& =\Lambda\left(x_{2 k}\right) d\left(x_{2 k}, x_{2 k+1}\right)+\Xi\left(x_{2 k}\right) d\left(x_{2 k+1}, x_{2 k+2}\right)\left(\frac{d\left(x_{2 k}, x_{2 k+1}\right)}{1+d\left(x_{2 k}, x_{2 k+1}\right)}\right) \\
& \precsim \Lambda\left(x_{2 k}\right) d\left(x_{2 k}, x_{2 k+1}\right)+\Xi\left(x_{2 k}\right) d\left(x_{2 k+1}, x_{2 k+2}\right) \\
& =\Lambda\left(T x_{2 k-1}\right) d\left(x_{2 k}, x_{2 k+1}\right)+\Xi\left(T x_{2 k-1}\right) d\left(x_{2 k+1}, x_{2 k+2}\right) \\
& \precsim \Lambda\left(x_{2 k-1}\right) d\left(x_{2 k}, x_{2 k+1}\right)+\Xi\left(x_{2 k-1}\right) d\left(x_{2 k+1}, x_{2 k+2}\right) \\
& =\Lambda\left(S x_{2 k-2}\right) d\left(x_{2 k}, x_{2 k+1}\right)+\Xi\left(S x_{2 k-2}\right) d\left(x_{2 k+1}, x_{2 k+2}\right) \\
& \precsim \Lambda\left(x_{2 k-2}\right) d\left(x_{2 k}, x_{2 k+1}\right)+\Xi\left(x_{2 k-2}\right) d\left(x_{2 k+1}, x_{2 k+2}\right) \\
& \vdots \\
& \precsim \Lambda\left(x_{0}\right) d\left(x_{2 k}, x_{2 k+1}\right)+\Xi\left(x_{0}\right) d\left(x_{2 k+1}, x_{2 k+2}\right),
\end{aligned}
$$

which is implies that

$$
d\left(x_{2 k+1}, x_{2 k+2}\right) \precsim\left(\frac{\Lambda\left(x_{0}\right)}{1-\Xi\left(x_{0}\right)}\right) d\left(x_{2 k}, x_{2 k+1}\right) .
$$

Similarly, we get

$$
\begin{aligned}
d\left(x_{2 k+2}, x_{2 k+3}\right) & =d\left(x_{2 k+3}, x_{2 k+2}\right) \\
& =d\left(S x_{2 k+2}, T x_{2 k+1}\right) \\
& \precsim \Lambda\left(x_{2 k+2}\right) d\left(x_{2 k+2}, x_{2 k+1}\right)+\frac{\Xi\left(x_{2 k+2}\right) d\left(x_{2 k+2}, S x_{2 k+2}\right) d\left(x_{2 k+1}, T x_{2 k+1}\right)}{1+d\left(x_{2 k+2}, x_{2 k+1}\right)} \\
& =\Lambda\left(x_{2 k+2}\right) d\left(x_{2 k+2}, x_{2 k+1}\right)+\frac{\Xi\left(x_{2 k+2}\right) d\left(x_{2 k+2}, x_{2 k+3}\right) d\left(x_{2 k+1}, x_{2 k+2}\right)}{1+d\left(x_{2 k+1}, x_{2 k+2}\right)} \\
& =\Lambda\left(x_{2 k+2}\right) d\left(x_{2 k+2}, x_{2 k+1}\right)+\Xi\left(x_{2 k+2}\right) d\left(x_{2 k+2}, x_{2 k+3}\right)\left(\frac{d\left(x_{2 k+2}, x_{2 k+1}\right)}{1+d\left(x_{2 k+1}, x_{2 k+2}\right)}\right) \\
& \precsim \Lambda\left(x_{2 n+2}\right) d\left(x_{2 k+2}, x_{2 k+1}\right)+\Xi\left(x_{2 k+2}\right) d\left(x_{2 k+2}, x_{2 k+3}\right) \\
& =\Lambda\left(T x_{2 k+1}\right) d\left(x_{2 k+2}, x_{2 k+1}\right)+\Xi\left(T x_{2 k+1}\right) d\left(x_{2 k+2}, x_{2 k+3}\right) \\
& \precsim \Lambda\left(x_{2 n+1}\right) d\left(x_{2 k+2}, x_{2 k+1}\right)+\Xi\left(x_{2 k+1}\right) d\left(x_{2 k+2}, x_{2 k+3}\right) \\
& =\Lambda\left(S x_{2 k}\right) d\left(x_{2 k+2}, x_{2 k+1}\right)+\Xi\left(S x_{2 k}\right) d\left(x_{2 k+2}, x_{2 k+3}\right) \\
& \precsim \Lambda\left(x_{2 k}\right) d\left(x_{2 k+2}, x_{2 k+1}\right)+\Xi\left(x_{2 k}\right) d\left(x_{2 k+2}, x_{2 k+3}\right) \\
& \vdots \\
& \precsim \Lambda\left(x_{0}\right) d\left(x_{2 k+2}, x_{2 k+1}\right)+\Xi\left(x_{0}\right) d\left(x_{2 k+2}, x_{2 k+3}\right) \\
& =\Lambda\left(x_{0}\right) d\left(x_{2 k+1}, x_{2 k+2}\right)+\Xi\left(x_{0}\right) d\left(x_{2 k+2}, x_{2 k+3}\right),
\end{aligned}
$$

which is implies that

$$
d\left(x_{2 k+2}, x_{2 k+3}\right) \precsim\left(\frac{\Lambda\left(x_{0}\right)}{1-\Xi\left(x_{0}\right)}\right) d\left(x_{2 k+1}, x_{2 k+2}\right) .
$$


Now, we set $\alpha:=\frac{\Lambda\left(x_{0}\right)}{1-\Xi\left(x_{0}\right)}$, it follows that

$$
\begin{aligned}
d\left(x_{n}, x_{n+1}\right) & \precsim \alpha d\left(x_{n-1}, x_{n}\right) \\
& \precsim \alpha^{2} d\left(x_{n-2}, x_{n-1}\right) \\
& \vdots \\
& \precsim \alpha^{n} d\left(x_{0}, x_{1}\right)
\end{aligned}
$$

for all $n \in \mathbb{N}$. Now, for any positive integer $m$ and $n$ with $m>n$, we have

$$
\begin{aligned}
d\left(x_{n}, x_{m}\right) & \precsim d\left(x_{n}, x_{n+1}\right)+d\left(x_{n+1}, x_{n+2}\right)+\cdots+d\left(x_{m-1}, x_{m}\right) \\
& \precsim \alpha^{n} d\left(x_{0}, x_{1}\right)+\alpha^{n+1} d\left(x_{0}, x_{1}\right)+\cdots+\alpha^{m-1} d\left(x_{0}, x_{1}\right) \\
& =\left(\alpha^{n}+\alpha^{n+1}+\cdots+\alpha^{m-1}\right) d\left(x_{0}, x_{1}\right) \\
& \precsim\left(\frac{\alpha^{n}}{1-\alpha}\right) d\left(x_{0}, x_{1}\right) .
\end{aligned}
$$

Therefore,

$$
\left|d\left(x_{n}, x_{m}\right)\right| \leq\left(\frac{\alpha^{n}}{1-\alpha}\right)\left|d\left(x_{0}, x_{1}\right)\right| .
$$

Since $\alpha \in[0,1)$, if we taking limit as $m, n \rightarrow 0$, then $\left|d\left(x_{n}, x_{m}\right)\right| \rightarrow 0$, which implies that $\left\{x_{n}\right\}$ is a Cauchy sequence. By completeness of $X$, there exists a point $z \in X$ such that $x_{k} \rightarrow z$ as $k \rightarrow \infty$. Next, we claim that $S z=z$. By the notion of a complex valued metric $d$, we have

$$
\begin{aligned}
d(z, S z) & \precsim d\left(z, x_{2 k+2}\right)+d\left(x_{2 k+2}, S z\right) \\
& =d\left(z, x_{2 k+2}\right)+d\left(T x_{2 k+1}, S z\right) \\
& =d\left(z, x_{2 k+2}\right)+d\left(S z, T x_{2 k+1}\right) \\
& \precsim d\left(x_{2 k+2}, z\right)+\Lambda(z) d\left(z, x_{2 k+1}\right)+\frac{\Xi(z) d(z, S z) d\left(x_{2 k+1}, T x_{2 k+1}\right)}{1+d\left(z, x_{2 k+1}\right)} \\
& =d\left(x_{2 k+2}, z\right)+\Lambda(z) d\left(z, x_{2 k+1}\right)+\frac{\Xi(z) d(z, S z) d\left(x_{2 k+1}, x_{2 k+2}\right)}{1+d\left(z, x_{2 k+1}\right)}
\end{aligned}
$$

which implies that

$$
|d(z, S z)| \leq\left|d\left(x_{2 k+2}, z\right)\right|+\Lambda(z)\left|d\left(z, x_{2 k+1}\right)\right|+\frac{\Xi(z)\left|d\left(x_{2 k+1}, x_{2 k+2}\right)\right||d(z, S z)|}{1+\left|d\left(z, x_{2 k+1}\right)\right|} .
$$

Taking $k \rightarrow \infty$, we have $|d(z, S z)|=0$, which implies that $d(z, S z)=0$. Thus, we get $z$ $=S z$. It follows similarly that $z=T z$. Therefore, $z$ is a common fixed point of $S$ and $T$.

Finally, we show that $z$ is a unique common fixed point of $S$ and $T$. Assume that there exists another common fixed point $z_{1}$ that is $z_{1}=S z_{1}=T z_{1}$. It follows from

$$
\begin{aligned}
d\left(z, z_{1}\right) & =d\left(S z, T z_{1}\right) \\
& \precsim \Lambda(z) d\left(z, z_{1}\right)+\frac{\Xi(z) d(z, S z) d\left(z_{1}, T z_{1}\right)}{1+d\left(z, z_{1}\right)} \\
& =\Lambda(z) d\left(z, z_{1}\right),
\end{aligned}
$$

that $\left|d\left(z, z_{1}\right)\right| \leq \Lambda(z)\left|d\left(z, z_{1}\right)\right|$. 
Since $\Lambda(z) \in[0,1)$, we have $\left|d\left(z, z_{1}\right)\right|=0$. Therefore, we have $z=z_{1}$ and thus $z$ is a unique common fixed point of $S$ and $T$.

Corollary 3.2. [[24], Theorem 4] Let $(X, d)$ be a complete complex valued metric space and $S, T: X \rightarrow X$. If $S$ and $T$ satisfy

$$
d(S x, T y) \precsim \lambda d(x, y)+\frac{\mu d(x, S x) d(y, T y)}{1+d(x, y)}
$$

for all $x, y \in X$, where $\lambda, \mu$ are nonnegative reals with $\lambda+\mu<1$. Then $S$ and $T$ have a unique common fixed point.

Proof. We can prove this result by applying Theorem 3.1 by setting $\Lambda(x)=\lambda$ and $\Xi(x)=\mu$.

Corollary 3.3. Let $(X, d)$ be a complete complex valued metric space and $T: X \rightarrow X$. If there exists a mapping $\Lambda, \Xi: X \rightarrow[0,1)$ such that for all $x, y \in X$ :

(i): $\Lambda(T x) \leq \Lambda(x)$ and $\Xi(T x) \leq \Xi(x)$;

(ii): $(\Lambda+\Xi)(x)<1$;

(iii): $d(T x, T y) \precsim \Lambda(x) d(x, y)+\frac{\Xi(x) d(x, T x) d(y, T y)}{1+d(x, y)}$.

Then $T$ has a unique fixed point.

Proof. We can prove this result by applying Theorem 3.1 with $S=T$.

Corollary 3.4. [[24], Corollary 5] Let $(X, d)$ be a complete complex valued metric space and $T: X \rightarrow X$. If $T$ satisfies

$$
d(T x, T y) \precsim \lambda d(x, y)+\frac{\mu d(x, T x) d(y, T y)}{1+d(x, y)}
$$

for all $x, y \in X$, where $\lambda, \mu$ are nonnegative reals with $\lambda+\mu<1$. Then $T$ has a unique fixed point.

Proof. We can prove this result by applying Corollary 3.3 with $\Lambda(x)=\lambda$ and $\Xi(x)=\mu$.

Theorem 3.5. Let $(X, d)$ be a complete complex valued metric space and $T: X \rightarrow X$. If there exists a mapping $\Lambda, \Xi: X \rightarrow[0,1)$ such that for all $x, y \in X$ and for some $n \in$ $\mathbb{N}$ :

(i): $\Lambda\left(T^{n} x\right) \leq \Lambda(x)$ and $\Xi\left(T^{n} x\right) \leq \Xi(x)$;

(ii): $(\Lambda+\Xi)(x)<1$;

(iii): $d\left(T^{n} x, T^{n} y\right) \precsim \Lambda(x) d(x, y)+\frac{\Xi(x) d\left(x, T^{n} x\right) d\left(y, T^{n} y\right)}{1+d(x, y)}$.

Then $T$ has a unique fixed point.

Proof. From Corollary 3.3, we get $T^{n}$ has a unique fixed point $z$. It follows from

$$
T^{n}(T z)=T\left(T^{n} z\right)=T z
$$

that $T z$ is a fixed point of $T^{n}$. Therefore $T z=z$ by the uniqueness of a fixed point of $T^{n}$ and then $z$ is also a fixed point of $T$. Since the fixed point of $T$ is also fixed point of $T^{n}$, the fixed point of $T$ is unique. 
Corollary 3.6. [[24], Corollary 6] Let $(X, d)$ be a complete complex valued metric space and $S, T: X \rightarrow X$. If T satisfy

$$
d\left(T^{n} x, T^{n} y\right) \precsim \lambda d(x, y)+\frac{\mu d\left(x, T^{n} x\right) d\left(y, T^{n} y\right)}{1+d(x, y)}
$$

for all $x, y \in X$ for some $n \in \mathbb{N}$, where $\lambda$, $\mu$ are nonnegative reals with $\lambda+\mu<1$. Then $T$ has a unique fixed point.

Proof. We can prove this result by applying Theorem 3.5 with $\Lambda(x)=\lambda$ and $\Xi(x)=\mu$.

Next, we prove a common fixed point theorem for weakly compatible mappings in complex valued metric spaces.

Theorem 3.7. Let $(X, d)$ be a complex valued metric space, $S, T: X \rightarrow X$ such that $T$ $(X) \subseteq S(X)$ and $S(X)$ is complete. If there exists two mappings $\Lambda, \Xi: X \rightarrow[0,1)$ such that for all $x, y \in X$ :

(i): $\Lambda(T x) \leq \Lambda(S x)$ and $\Xi(T x) \leq \Xi(S x)$;

(ii): $(\Lambda+\Xi)(S x)<1$

(iii): $d(T x, T y) \precsim \Lambda(S x) d(S x, S y)+\frac{\Xi(S x) d(S x, T x) d(S y, T y)}{1+d(S x, S y)}$.

Then $S$ and $T$ have a unique point of coincidence in $X$. Moreover, if $S$ and $T$ are weakly compatible, then $S$ and $T$ have a unique common fixed point in $X$.

Proof. By Lemma 2.14, there exists $E \subseteq X$ such that $S(E)=S(X)$ and $S: E \rightarrow X$ is one-to-one. Since

$$
T(E) \subseteq T(X) \subseteq S(X)=S(E),
$$

we can define a mapping $\Theta: S(E) \rightarrow S(E)$ by

$$
\Theta(S x)=T x .
$$

Since $S$ is one-to-one on $E$, then $\Theta$ is well-defined. From (i) and (3.15), we have

$$
\Lambda(\Theta(S x)) \leq \Lambda(S x) \text { and } \Xi(\Theta(S x)) \leq \Xi(S x) .
$$

From (iii) and (3.15), we get

$$
d(\Theta(S x), \Theta(S y)) \precsim \Lambda(S x) d(S x, S y)+\frac{\Xi(S x) d(S x, \Theta(S x)) d(S y, \Theta(S y))}{1+d(S x, S y)}
$$

for all $S x, S y \in S(E)$. From $S(E)=S(X)$ is complete and (3.16) and (3.17) are holds, we use Corollary 3.3 with a mapping $\Theta$, then there exists a unique fixed point $z \in S(X)$ such that $\Theta z=z$. Since $z \in S(X)$, we have $z=S w$ for some $w \in X$. So $\Theta(S w)=S w$ that is $T w=S w$. Therefore, $T$ and $S$ have a unique point of coincidence.

Next, we claim that $S$ and $T$ have a common fixed point. Since $S$ and $T$ are weakly compatible and $z=T w=S w$, we get

$$
S z=S T w=T S w=T z .
$$

Hence $S z=T z$ is a point of coincidence of $S$ and $T$. Since $z$ is the only point of coincidence of $S$ and $T$, we get $z=S z=T z$ which implies that $z$ is a common fixed point of $S$ and $T$. 
Finally, we show that $z$ is a unique common fixed point of $S$ and $T$. Assume that $t$ be another common fixed point that is

$$
t=S t=T t
$$

Thus $t$ is also a point of coincidence of $S$ and $T$. However, we know that $z$ is a unique point of coincidence of $S$ and $T$. Therefore, we get $t=z$ that is $z$ is a unique common fixed point of $S$ and $T$.

\section{Applications}

In this section, we apply Theorem 3.1 to the existence of common solution of the system of Urysohn integral equations.

Theorem 4.1. Let $X=C\left([a, b], \mathbb{R}^{n}\right)$, where $[a, b] \subseteq \mathbb{R}^{+}$and $d: X \times X \rightarrow \mathbb{C}$ is define by

$$
d(x, y)=\max _{t \in[a, b]}\|x(t)-y(t)\|_{\infty} \sqrt{1+a^{2}} e^{i \tan ^{-1} a}
$$

Consider the Urysohn integral equations

$$
\begin{aligned}
& x(t)=\int_{a}^{b} K_{1}(t, s, x(s)) d s+g(t), \\
& x(t)=\int_{a}^{b} K_{2}(t, s, x(s)) d s+h(t),
\end{aligned}
$$

where $t \in[a, b] \subset \mathbb{R}$ and $x, g, h \in X$.

Suppose that $K_{1}, K_{2}:[a, b] \times[a, b] \times \mathbb{R}^{n} \rightarrow \mathbb{R}^{n}$ are such that $F_{x}, G_{x} \in X$ for all $x \in$ $X$, where

$$
F_{x}(t)=\int_{a}^{b} K_{1}(t, s, x(s)) d s
$$

and

$$
G_{x}(t)=\int_{a}^{b} K_{2}(t, s, x(s)) d s
$$

for all $t \in[a, b]$.

If there exists two mappings $\Lambda, \Xi: X \rightarrow[0,1)$ such that for all $x, y \in X$ the following holds:

(i) $\Lambda\left(F_{x}+g\right) \leq \Lambda(x)$ and $\Xi\left(F_{x}+g\right) \leq \Xi(x)$;

(ii) $\Lambda\left(G_{x}+h\right) \leq \Lambda(x)$ and $\Xi\left(G_{x}+h\right) \leq \Xi(x)$;

(iii) $(\Lambda+\Xi)(x)<1$;

(iv) $\left\|F_{x}(t)-G_{y}(t)+g(t)-h(t)\right\|_{\infty} \sqrt{1+a^{2}} e^{i \tan ^{-1} a} \precsim \Lambda(x) A(x, y)(t)+\Xi(x) B(x, y)(t)$, where $A(x, y)(t)=\|x(t)-y(t)\|_{\infty} \sqrt{1+a^{2}} e^{i \tan ^{-1} a}$, 


$$
B(x, y)(t)=\frac{\left\|F_{x}(t)+g(t)-x(t)\right\|_{\infty}\left\|G_{y}(t)+h(t)-y(t)\right\|_{\infty}}{1+d(x, y)} \sqrt{1+a^{2}} e^{i \tan ^{-1} a},
$$

then the system of integral Equations (4.1) and (4.2) have a unique common solution.

Proof. It is easily to check that $(X, d)$ is a complex valued metric space. Define two mappings $S, T: X \times X \rightarrow X$ by $S x=F_{x}+g$ and $T x=G_{x}+h$. Then

$$
\begin{gathered}
d(S x, T y)=\max _{t \in[a, b]}\left\|F_{x}(t)-G_{y}(t)+g(t)-h(t)\right\|_{\infty} \sqrt{1+a^{2}} e^{i \tan ^{-1} a}, \\
d(x, S x)=\max _{t \in[a, b]}\left\|F_{x}(t)+g(t)-x(t)\right\|_{\infty} \sqrt{1+a^{2}} e^{i \tan ^{-1} a}
\end{gathered}
$$

and

$$
d(y, T y)=\max _{t \in[a, b]}\left\|G_{y}(t)+h(t)-\gamma(t)\right\|_{\infty} \sqrt{1+a^{2}} e^{i \tan ^{-1} a} .
$$

It is easily seen that for all $x, y \in X$, we have

(i) $\Lambda(S x) \leq \Lambda(x)$ and $\Xi(S x) \leq \Xi(x)$;

(ii) $\Lambda(T x) \leq \Lambda(x)$ and $\Xi(T x) \leq \Xi(x)$;

(iii) $d(S x, T y) \precsim \Lambda(x) d(x, y)+\frac{\Xi(x) d(x, S x) d(y, T y)}{1+d(x, y)}$.

By Theorem 3.1, we get $S$ and $T$ have a common fixed point. Thus there exists a unique point $x \in X$ such that $x=S x=T x$. Now, we have

$$
x=S x=F_{x}+g
$$

and

$$
x=T x=G_{x}+h
$$

that is

$$
x(t)=\int_{a}^{b} K_{1}(t, s, x(s)) d s+g(t)
$$

and

$$
x(t)=\int_{a}^{b} K_{2}(t, s, x(s)) d s+h(t) .
$$

Therefore, we can conclude that the Urysohn integral (4.1) and (4.2) have a unique com mon fixed point

\section{Conclusion}

In this article, we modified and generalized a contraction mapping of Azam et al. [24] and proved some fixed point and common fixed point theorems for new generalization contraction mappings in a complex valued metric space. Although, Theorem 1.1 of Azam et al. [24] is an essential tool in the complex valued metric space to claim the existence of common fixed points of some mappings. However, it is the most 
interesting to define such mappings $\Lambda$ and $\Xi$ as another auxiliary tool to claim the existence of a fixed point. In fact, all the main results in this article are some of choices for solving problems in a complex valued metric space. Our results may be the motivation to other authors for extending and improving these results to be suitable tools for their applications.

\section{Acknowledgements}

The authors would like to express his sincere thanks to the anonymous referee for their valuable comments and useful suggestions in improving the manuscript. W. Sintunavarat would like to thank the Research Professional Development Project Under the Science Achievement Scholarship of Thailand (SAST) and the Faculty of Science, KMUTT for financial support during the preparation of this manuscript for the Ph.D. Program at KMUTT. This research was supported by the Higher Education Research Promotion and National Research University Project of Thailand, Office of the Higher Education Commission (NRU-CSEC No. 54000267).

\section{Authors' contributions}

WS designed and performed all the steps of proof in this research and also wrote the paper. PK participated in the design of the study and suggest many good ideas that made this paper possible and helped to draft the first manuscript. All authors read and approved the final manuscript.

\section{Competing interests}

The authors declare that they have no competing interests.

Received: 21 January 2012 Accepted: 13 April 2012 Published: 13 April 2012

\section{References}

1. Banach, S: Sur les opérations dans les ensembles abstraits et leurs applications aux équations intégrales. Fund Math. 3, 133-181 (1922)

2. Abbas, M, Cho, YJ, Nazir, T: Common fixed point theorems for four mappings in TVS-valued cone metric spaces. J Math Inequal. 5, 287-299 (2011)

3. Cho, YJ: Fixed points for compatible mappings of type (A). Math Japon. 18, 497-508 (1993)

4. Cho, YJ, Saadati, R, Wang, S: Common fixed point theorems on generalized distance in order cone metric spaces. Comput Math Appl. 61, 1254-1260 (2011). doi:10.1016/j.camwa.2011.01.004

5. Graily, E, Vaezpour, SM, Saadati, R, Cho, YJ: Generalization of fixed point theorems in ordered metric spaces concerning generalized distance. Fixed Point Theory Appl. 2011, 30 (2011). doi:10.1186/1687-1812-2011-30

6. Kaewkhao, A, Sintunavarat, W, Kumam, P: Common fixed point theorems of c-distance on cone metric spaces. J Nonlinear Anal Appl. 2012, 11 (2012)

7. Marsh, MM: Fixed point theorems for partially outward mappings. Topol Appl. 153, 3546-3554 (2006). doi:10.1016/j. topol.2006.03.019

8. Mongkolkeha, C, Sintunavarat, W, Kumam, P: Fixed point theorems for contraction mappings in modular metric spaces. Fixed Point Theory Appl. 2011, 93 (2011). doi:10.1186/1687-1812-2011-93

9. Marsh, MM, Prajs, JR: Brush spaces and the fixed point property. Topol Appl. 158, 1085-1089 (2011). doi:10.1016/j. topol.2011.03.004

10. Shahzad, N: Fixed point results for multimaps in CAT(0) spaces. Topol Appl. 156, 997-1001 (2009). doi:10.1016/j. topol.2008.11.016

11. Sintunavarat, W, Kumam, P: Weak condition for generalized multi-valued $(f, a, \beta)$-weak contraction mappings. Appl Math Lett. 24, 460-465 (2011). doi:10.1016/j.aml.2010.10.042

12. Sintunavarat, W, Kumam, P: Gregus type fixed points for a tangential multi-valued mappings satisfying contractive conditions of integral type. J Inequal Appl. 2011, 3 (2011). doi:10.1186/1029-242X-2011-3

13. Sintunavarat, W, Kumam, P: Common fixed point theorems for hybrid generalized multi-valued contraction mappings. Appl Math Lett. 25, 52-57 (2012). doi:10.1016/j.aml.2011.05.047

14. Sintunavarat, $W$, Kumam, $P$ : Common fixed point theorems for generalized $\mathcal{J} \mathcal{H}$-operator classes and invariant approximations. J Inequal Appl. 2011, 67 (2011). doi:10.1186/1029-242X-2011-67

15. Sintunavarat, W, Kumam, P: Common fixed point theorem for cyclic generalized multi-valued contraction mappings. Appl Math Lett. (2012, in press)

16. Sintunavarat, W, Kumam, P: Common fixed points for R-weakly commuting in fuzzy metric spaces. Annali dell'Universita di Ferrara. (2012, in press)

17. Zhao, X: Fixed point classes on symmetric product spaces. Topol Appl. 157, 1859-1871 (2010). doi:10.1016/j. topol.2010.02.022

18. Kirk, WA: Some recent results in metric fixed point theory. J Fixed Point Theory Appl. 2, 195-207 (2007). doi:10.1007/ s11784-007-0031-8

19. Semple, C, Steel, M: Phylogenetics, Oxford Lecture Ser. In Math Appl, vol. 24,Oxford Univ. Press, Oxford (2003)

20. Perov, Al: On the Cauchy problem for a system of ordinary difierential equations. Pvi-blizhen Met Reshen Diff Uvavn. 2, 115-134 (1964)

21. Mustafa, Z, Sims, B: A new approach to generalized metric spaces. J Nonlinear Convex Anal. 7(2):289-297 (2006)

22. Huang, LG, Zhang, X: Cone metric spaces and fixed point theorems of contractive mappings. J Math Anal Appl. 332, 1468-1476 (2007). doi:10.1016/j.jmaa.2005.03.087

23. Chistyakov, W: Modular metric spaces, I: basic concepts. Nonlinear Anal. 72, 1-14 (2010). doi:10.1016/j.na.2009.04.057 
24. Azam, A, Brian, F, Khan, M: Common Fixed Point Theorems in Complex Valued Metric Spaces. Numer Funct Anal Optim. 32(3):243-253 (2011). doi:10.1080/01630563.2011.533046

25. Jungck, G: Commuting maps and fixed points. Am Math Monthly. 83, 261-263 (1976). doi:10.2307/2318216

26. Sessa, S: On a weak commutativity condition of mappings in fixed point consideration. Publ Inst Math. 32(46):149-153 (1982)

27. Jungck, G: Compatible mappings and common fixed points. Int J Math Math Sci. 9, $771-779$ (1986). doi:10.1155/ S0161171286000935

28. Jungck, G: Compatible mappings and common fixed points (2). Int J Math Math Sci. 11, $285-288$ (1988). doi:10.1155/ S0161171288000341

29. Jungck, G: Common fixed points of commuting and compatible maps on compacta. Proc Am Math Soc. 103, 977-983 (1988). doi:10.1090/S0002-9939-1988-0947693-2

30. Jungck, G: Common fixed points for non-continuous non-self mappings on a non-numeric spaces. Far East J Math Sci. 4(2):199-212 (1996)

31. Djoudi, A, Nisse, L: Gregus type fixed points for weakly compatible maps. Bull Belg Math Soc Simon Stevin. 10(3):369-378 (2003)

32. Haghi, RH, Rezapour, Sh, Shahzadb, N: Some fixed point generalizations are not real generalizations. Nonlinear Anal. 74 1799-1803 (2011). doi:10.1016/.na.2010.10.052

doi:10.1186/1029-242X-2012-84

Cite this article as: Sintunavarat and Kumam: Generalized common fixed point theorems in complex valued metric spaces and applications. Journal of Inequalities and Applications 2012 2012:84.

\section{Submit your manuscript to a SpringerOpen ${ }^{\odot}$} journal and benefit from:

- Convenient online submission

Rigorous peer review

- Immediate publication on acceptance

- Open access: articles freely available online

- High visibility within the field

- Retaining the copyright to your article

Submit your next manuscript at $\boldsymbol{s p r i n g e r o p e n . c o m ~}$ 